\title{
An overview of the 6th World Symposium on Pulmonary Hypertension
}

\author{
Nazzareno Galiè ${ }^{1}$, Vallerie V. McLaughlin², Lewis J. Rubin ${ }^{3}$ and \\ Gerald Simonneau ${ }^{4,5}$
}

Affiliations: ${ }^{1}$ Dept of Experimental, Diagnostic and Specialty Medicine-DIMES, Alma Mater Studiorum, University of Bologna, Bologna, Italy. ${ }^{2}$ The University of Michigan, Cardiovascular Medicine, Ann Arbor, MI, USA. ${ }^{3}$ University of California, San Diego, School of Medicine, La Jolla, CA, USA. ${ }^{4}$ Univ. Paris-Sud, AP-HP, Centre de Référence de l'Hypertension Pulmonaire Sévère, Service de Pneumologie, Département HospitaloUniversitaire (DHU) Thorax Innovation (TORINO), Hôpital de Bicêtre, Le Kremlin Bicêtre, France. ${ }^{5}$ INSERM UMR_S999, LabEx LERMIT, Hôpital Marie Lannelongue, Le Plessis Robinson, France.

Correspondence: Nazzareno Galiè, Dept of Experimental, Diagnostic and Specialty Medicine (DIMES), Alma Mater Studiorum, University of Bologna, Via Massarenti 9, 40138 Bologna, Italy. E-mail: nazzareno.galiedunibo.it

@ERSpublications

State of the art summary on diagnosis, prognosis, therapy and future perspectives of pulmonary hypertension http://ow.ly/8MHN30mGtqs

Cite this article as: Galiè N, McLaughlin VV, Rubin LJ, et al. An overview of the 6th World Symposium on Pulmonary Hypertension. Eur Respir J 2019; 53: 1802148 [https://doi.org/10.1183/13993003.02148-2018].

Since 1973 the World Symposia on Pulmonary Hypertension (WSPH) proceedings have summarised the scientific advances and future needs in this field through the efforts of multiple task forces, each focusing on a different aspect of pulmonary hypertension (PH) [1]. The 6th WSPH comprised 124 experts, divided into 13 task forces, that began their work in January 2017 and presented their consensus opinions to an audience of 1376 participant attendees between February 27 and March 1, 2018 in Nice, France. A newly created task force dedicated to patients' perspectives, including representatives of patients' associations worldwide, was added for the 6th WSPH.

The task forces continued their work after the 6th WSPH to incorporate participants' input during the plenary session discussions into the 13 manuscripts published in the current issue of the European Respiratory Journal. The publication process, which in the past had been performed after each world symposium and included editors and blinded peer-review and revisions, has been modified with these proceedings in order to improve the quality and the timeliness of these state-of-the art articles. The key points included in each task force's manuscript, representing both current achievements and starting points for future research in the field of the pulmonary vascular science, are summarised below.

The task force on pathology and pathobiology summarised the most advanced achievements in the cellular and molecular basis and pathology of pulmonary vascular remodelling associated with various forms of $\mathrm{PH}$ [2]. The manuscript reports new insights on specific pulmonary vascular lesions, including plexiform lesions, complex lesions and venous and venular lesions. Recent advances in cellular abnormalities are discussed, such as dysfunction of the pulmonary vascular endothelium, accumulation of pulmonary artery smooth muscle cells and adventitial fibroblasts, and dysregulation of the innate and adaptive immune system. Finally, multiple molecular mechanisms are explored and considered as emerging therapeutic targets that may constitute the rationale for future clinical studies.

The genetics and genomics task force estimated that about $25-30 \%$ of patients diagnosed with idiopathic pulmonary arterial hypertension have an underlying Mendelian genetic cause for their condition and

Received: Nov 102018 | Accepted: Nov 132018

Copyright $\odot$ ERS 2019. This article is open access and distributed under the terms of the Creative Commons Attribution Non-Commercial Licence 4.0. 
should be classified as heritable pulmonary arterial hypertension [3]. The article reports links between mutations and disease, and factors affecting disease penetrance. Genetic counselling and testing, management of healthy mutation carriers and psychosocial considerations and reproductive options are also discussed. Advances in DNA sequencing technology may facilitate genomic studies in large cohorts and potentially lead to a molecular classification of the disease. This may help identify novel targets for new drugs, expanding our therapeutic options.

The task force on the pathophysiology of the right ventricle and of the pulmonary circulation has revisited the pathophysiological description of the cardio-pulmonary unit (consisting of both the right ventricle and the pulmonary vascular system) in order to improve clinical data interpretation [4]. A better understanding of right heart haemodynamic data as well as imaging data of the right ventricle obtained by echocardiography or magnetic resonance imaging is also provided. Provocative tests such as exercise and fluid loading are addressed, and the need for additional outcome studies to clarify the clinical relevance of an "abnormal" response is outlined. Finally, an update is provided on the latest insights in the pathobiology of right ventricular failure, including key pathways of molecular adaptation of the pressure-overloaded right ventricle.

One of the most significant (and controversial) recommendations from the 6th WSPH has been the proposal by the task force on haemodynamic definitions and clinical classification, to reconsider the haemodynamic definition of $\mathrm{PH}$ [5]. Based on data from normal subjects, the normal mean pulmonary arterial pressure (mPAP) at rest is approximately $14.0 \pm 3.3 \mathrm{mmHg}$ [6]. Two standard deviations above this mean value would indicate that a mPAP $>20 \mathrm{mmHg}$ is the threshold for abnormal pulmonary arterial pressure (above the 97.5th percentile). However, this level of mPAP is not sufficient to define pulmonary vascular disease, since it could be due to increases in cardiac output or pulmonary artery wedge pressure (PAWP). The task force has therefore proposed including a pulmonary vascular resistance (PVR) $\geqslant 3 \mathrm{WU}$ into the definition of pre-capillary $\mathrm{PH}$ associated with $\mathrm{mPAP}>20 \mathrm{mmHg}$, irrespective of aetiology. Future trials should assess the efficacy of pulmonary arterial hypertension (PAH) medications (currently approved based on a mPAP $\geqslant 25 \mathrm{mmHg}$ ) in patients with mPAP from 21 to $24 \mathrm{mmHg}$ and PVR $\geqslant 3 \mathrm{WU}$. Due to limited data, the task force declined to identify a clinically useful definition of exercise $\mathrm{PH}$, encouraging additional outcome studies instead. The clinical classification of $\mathrm{PH}$ was simplified, maintaining the traditional five-group structure. A new entity entitled "PAH long-term responders to calcium channel blockers" was introduced to group 1.

The task force on $\mathrm{PH}$ diagnosis has revised the diagnostic algorithm, providing a methodical approach for diagnosis of patients with suspected PH both prior to and following referral to expert centres [7]. In addition, expedited referral has been recommended for high-risk or complex patients, as well as patients with confounding comorbidities. Updated screening procedures in asymptomatic patients with conditions associated with a high $\mathrm{PH}$ prevalence have been proposed, together with a review of current diagnostic tools and emerging diagnostic technologies.

The task force on clinical risk stratification and medical therapy in PAH patients has provided an update of all data available on these topics [8]. The strong relationship between risk stratification, initial treatment strategy and follow-up treatment escalations has been emphasised and serves as the rationale for a treatment strategy that is based on disease severity as assessed by a multi-parametric risk stratification approach. Clinical, exercise, right ventricular function and haemodynamic parameters and biomarkers are combined to define a low-, intermediate- or high-risk status based on the expected 1-year mortality. The comprehensive treatment algorithm provides an overview of initial strategies, including monotherapy (in a minority of patients), and double or triple combination therapy. Further treatment escalation is required in case low-risk status (considered as treatment goal) is not achieved in structured follow-up assessments.

The task force on right ventricular assistance and lung transplantation has presented a comprehensive intensive care approach for patients with $\mathrm{PH}$ and right-sided heart failure, including treatment of factors causing or contributing to heart failure, careful fluid management and strategies to reduce right ventricular afterload and improve cardiac function [9]. Extracorporeal membrane oxygenation should be considered in candidates for lung transplantation (bridge to transplant) or, occasionally, in patients with a reversible cause of right-sided heart failure (bridge to recovery). For patients with advanced disease, lung transplantation remains an important treatment option. Patients should be referred to a transplant centre when they remain in an intermediate- or high-risk category despite receiving maximal PAH therapy. In experienced centres, the 1-year survival rates after lung transplantation for $\mathrm{PH}$ now exceeds $90 \%$.

The task force on clinical trial design and new therapies for PAH has reviewed the progress in clinical trial design and end-points achieved over the past two decades, highlighting the crucial collaboration between international experts, industry and regulatory agencies [10]. New drug targets have been proposed and are summarised, including genetics, epigenetics, DNA damage, growth factors, metabolism, inflammation and immune modulation, oestrogen signalling, oxidative and hypoxic stress, serotonin and humoral 
modulation. Additional strategies such as pulmonary artery denervation and stem cell therapies are also discussed. The task force also addressed the challenges facing future PAH clinical trials and has outlined the characteristics for phase 1,2 and 3 registration studies.

New defining criteria for the different haemodynamic types of PH that occur with left heart disease (LHD) has been proposed by the task force on $\mathrm{PH}$ due to left heart disease [11]. After consideration of the changes in the general definition of $\mathrm{PH}$ [5], the proposed haemodynamic definition of $\mathrm{PH}$ in LHD was: 1) isolated post-capillary PH: PAWP $>15 \mathrm{mmHg}$ and $\mathrm{mPAP}>20 \mathrm{mmHg}$ and PVR $<3 \mathrm{WU}$; 2) combined post- and precapillary PH: PAWP $>15 \mathrm{mmHg}$ and $\mathrm{mPAP}>20 \mathrm{mmHg}$ and PVR $\geqslant 3 \mathrm{WU}$. The importance of the differential diagnosis between idiopathic $\mathrm{PAH}$ and $\mathrm{PH}$ due to heart failure with preserved left ventricular ejection fraction has been emphasised. A pre-test probability score for PH-LHD may help in the differential diagnosis. The nomenclature of PAH with cardiovascular risk factors should be preferred over any other, to account for their co-existence without suggesting that these risk factors are de facto the cause of the pulmonary vascular disease. The diagnostic relevance of provocative tests such as fluid loading and exercise were also discussed, together with the uncertainties over their clinical meaning. Finally, since multicentre randomised trials using PAH therapies in PH-LHD have not demonstrated benefit and have raised safety concerns, their use is still not recommended by the task force in PH-LHD.

The task force on $\mathrm{PH}$ due to chronic lung disease points out that $\mathrm{PH}$ frequently complicates the course of patients with various forms of chronic lung disease (CLD-PH) [12]. CLD-PH is invariably associated with reduced functional ability, impaired quality of life, greater oxygen requirements and an increased risk of mortality. The different aetiologies are discussed and the haemodynamic profile is compared with the combination of physiological and imaging assessment. An updated definition and severity grading of CLD-PH is also provided. The risk-to-benefit ratio of PAH approved drugs in CLD-PH patients has been revisited in depth. Although such therapy cannot be endorsed given the current lack of efficacy evidence and in the face of serious safety concerns, future studies in this area are strongly encouraged.

The task force on chronic thromboembolic pulmonary hypertension (CTEPH) has provided an overview of the state-of-the-art diagnostic and treatment modalities in this rapidly evolving field [13]. The new entity of chronic thromboembolic disease (CTED) has been characterised by similar symptoms and perfusion defects to CTEPH, but without $\mathrm{PH}$ at rest. CTEPH treatment recommendations should not yet be applied to CTED, since additional prospective studies are needed. Digital subtraction pulmonary angiography had been considered the gold standard for characterising vessel morphology in CTEPH, but is being challenged by advances in noninvasive modalities. Computed tomography pulmonary angiography is currently widely used for assessment of operability. Pulmonary endarterectomy (PEA) is the treatment of choice, and patient operability should be evaluated by an expert multidisciplinary CTEPH team including a PEA surgeon, $\mathrm{PH}$ expert, balloon pulmonary angioplasty (BPA) interventionist and radiologist. BPA, an emerging procedure in non-operable patients, requires specific training of dedicated interventionists. The level of expertise of the CTEPH team should be evaluated based on the centre's number of procedures performed and the outcome results. Medical therapy has a supporting role to improve symptoms and haemodynamics.

The task force on paediatric $\mathrm{PH}$ has revised a number of aspects of this specific field [14]. The definition of $\mathrm{PH}$ is consistent with the newly proposed definition in adults [5]. The use of cardiac catheterisation as a diagnostic modality and haemodynamic assessment of $\mathrm{PAH}$, including acute vasoreactivity testing, are addressed. The common clinical classification for both adults and children has been integrated with modifications in order to highlight aspects of paediatric disorders and better address specific features of paediatric $\mathrm{PH}$ within the core of the existing classification. Several features of paediatric $\mathrm{PH}$, including the prominence of neonatal $\mathrm{PAH}$, especially in preterm infants and those with developmental lung diseases and novel genetic causes of paediatric PAH, are highlighted. Although a lack of clinical trial data for the use of PAH-targeted therapy in this population persists, emerging data are improving the identification of appropriate targets for goal-oriented therapy in children. Such data will likely improve future clinical trial design to enhance outcomes in paediatric PAH. Specific paediatric interventional management of PAH, including the Potts shunt, were also discussed.

The importance of the patients' perspective has been outlined by a newly created task force [15]. Despite recent progress in patients' involvement in the $\mathrm{PH}$ field, patient surveys indicate that more can be done to improve this aspect. The relevance of health-related quality of life has been outlined, and specific questionnaire use may improve direct clinical care. Patients should be provided with access to accredited specialist centres that provide a multidisciplinary approach including narrative medicine, shared decision making, palliative care and participation in education. The role of patients' associations in supporting patients and carers, lobbying for access to best care and treatments, providing input in to the development of clinical trials and registries, and focusing on the patient perspective have also been highlighted. 
Conflict of interest: N. Galiè reports grants and personal fees from Actelion, Bayer, GSK and Pfizer, and personal fees from MSD, outside the submitted work. V.V. McLaughlin reports grants and personal fees from Actelion, Acceleron, Arena and Bayer, personal fees from Caremark and United Therapeutics, and grants from Gilead and Sonovie, during the conduct of the study. L. Rubin reports personal fees from Actelion, Arena, Bellerophon, SoniVie and Roivant, during the conduct of the study. G. Simonneau reports grants, personal fees and non-financial support from Actelion Pharmaceuticals, Bayer Healthcare, Merck and GlaxoSmithKline.

\section{References}

1 Galiè N, Simonneau G. The Fifth World Symposium on Pulmonary Arterial Hypertension. J Am Coll Cardiol 2013; 62: Suppl. 25, D1-D3.

2 Humbert M, Guignabert C, Bonnet S, et al. Pathology and pathobiology of pulmonary hypertension: state of the art and research perspectives. Eur Respir J 2019; 53: 1801887.

3 Morrell NW, Aldred MA, Chung WK, et al. Genetics and genomics of pulmonary arterial hypertension. Eur Respir J 2019; 53: 1801899.

4 Vonk Noordegraaf A, Chin KM, Haddad F, et al. Pathophysiology of the right ventricle and of the pulmonary circulation in pulmonary hypertension: an update. Eur Respir J 2019; 53: 1801900.

5 Simonneau G, Montani D, Celermajer DS, et al. Haemodynamic definitions and updated clinical classification of pulmonary hypertension. Eur Respir J 2019; 53: 1801913.

6 Kovacs G, Berghold A, Scheidl S, et al. Pulmonary arterial pressure during rest and exercise in healthy subjects A systematic review. Eur Respir J 2009; 34: 888-894.

7 Frost A, Badesch D, Gibbs JSR, et al. Diagnosis of pulmonary hypertension. Eur Respir J 2019; 53: 1801904.

8 Galiè N, Channick RN, Frantz RP, et al. Risk stratification and medical therapy of pulmonary arterial hypertension. Eur Respir J 2019; 53: 1801889.

9 Hoeper MM, Benza RL, Corris P, et al. Intensive care, right ventricular support and lung transplantation in patients with pulmonary hypertension. Eur Respir J 2019; 53: 1801906.

10 Sitbon O, Gomberg-Maitland M, Granton J, et al. Clinical trial design and new therapies for pulmonary arterial hypertension. Eur Respir J 2019; 53: 1801908.

11 Vachiéry J-L, Tedford RJ, Rosenkranz S, et al. Pulmonary hypertension due to left heart disease. Eur Respir J 2019; 53: 1801897.

12 Nathan SD, Barbera JA, Gaine SP, et al. Pulmonary hypertension in chronic lung disease and hypoxia. Eur Respir J 2019; 53: 1801914

13 Kim NH, Delcroix M, Jais X, et al. Chronic thromboembolic pulmonary hypertension. Eur Respir J 2019; 53: 1801915.

14 Rosenzweig EB, Abman SH, Adatia I, et al. Paediatric pulmonary arterial hypertension: updates on definition, classification, diagnostics and management. Eur Respir J 2019; 53: 1801916.

15 McGoon MD, Ferrari P, Armstrong I, et al. The importance of patient perspectives in pulmonary hypertension. Eur Respir J 2019; 53: 1801919. 